\title{
Retrospective study on the effects of the prognosis of patients treated with extracorporeal membrane oxygenation combined with continuous renal replacement therapy
}

\author{
Ping $\mathrm{He}^{1}$, Shixin Zhang ${ }^{1}$, Bingyang $\mathrm{Hu}^{2}$, Wei $\mathrm{Wu}^{1}$ \\ ${ }^{1}$ Cardiothoracic Surgery Department, Southwest Hospital, The Third Military Medical University, Chongqing 400038, China; ${ }^{2}$ Department of \\ Clinical Medicine, North Sichuan Medical College, Nanchong 637000, China \\ Contributions: (I) Conception and design: P He, W Wu; (II) Administrative support: P He, S Zhang, W Wu; (III) Provision of study materials or \\ patients: P He, S Zhang, W Wu; (IV) Collection and assembly of data: S Zhang, B Hu; (V) Data analysis and interpretation: P He, B Hu, W Wu; (VI) \\ Manuscript writing: All authors; (VII) Final approval of manuscript: All authors. \\ Correspondence to: Wei Wu. Cardiothoracic Surgery Department, Southwest Hospital, The Third Military Medical University, Chongqing 400038, \\ China. Email: wuweiyahoo@sohu.com.
}

Background: Patients undergoing extracorporeal membrane oxygenation (ECMO) treatment often have severe fluid overload and electrolyte imbalances and may even suffer acute kidney injury (AKI). It is often necessary to use continuous renal replacement therapy (CRRT). In this study, we aimed to retrospectively analyze the prognosis of patients treated with ECMO combined with CRRT and to find the independent factors that affect the survival rate.

Methods: There were 32 patients who were treated with ECMO combined with CRRT in our hospital from January 2007 to December 2017 who were analyzed. All of the patients were divided into a survival group and death group. The clinical indicators and biochemical indexes of the two groups were observed, and their differences were compared. Multivariate logistic regression analysis was carried out to determine the independent risk factors.

Results: The fluid balance at ECMO day 3, SOFA score and lactate at CRRT initiation, sequential organ failure assessment (SOFA) score at ECMO weaning, CRRT duration, ECMO to CRRT interval, mechanical ventilation (MV) duration, length of ICU, and overall hospital length of stay were statistically significant $(\mathrm{P}<0.05)$. The clinical biochemical indexes at CRRT initiation and ECMO weaning [serum creatinine, $\mathrm{pH}$, white blood cell (WBC), hemoglobin $(\mathrm{Hb})$, bilirubin]; patient's age, gender and $\mathrm{BMI}$; and the fluid balance at ECMO days 1 and 7 were not statistically significance $(\mathrm{P}>0.05)$. The fluid balance at ECMO day 3 and lactate at CRRT initiation by multivariable logistic regression analysis were independent risk factors affecting patient prognosis.

Conclusions: The fluid balance at ECMO day 3 and lactate at CRRT initiation are the prognosis independent risk factors for ECMO + CRRT patients.

Keywords: Extracorporeal membrane oxygenation (ECMO); continuous renal replacement therapy (CRRT); prognosis

Submitted Jul 03, 2018. Accepted for publication Nov 02, 2018.

doi: $10.21037 /$ atm.2018.11.12

View this article at: http://dx.doi.org/10.21037/atm.2018.11.12 


\section{Introduction}

In recent years, the number of patients with life-threatening heart or lung failure treated with extracorporeal membrane oxygenation (ECMO) has been increasing. About 30-50\% of patients with ECMO have acute kidney injury (AKI). Although continuous renal replacement therapy (CRRT) provides an effective treatment, studies have found that the 98-day survival rate of ECMO patients with AKI is only $17 \%$ (1). CRRT using during ECMO is an independent risk factor related to ECMO weaning failure (2) and the prediction (3). The indication of ECMO and CRRT combination has not been clearly defined. Most studies are based on pediatric data, and adult data are insufficient. Moreover, ECMO patients are in a critical condition, and some patients are not up to the KDIGO classification standards for AKI (for example, their SCr value may not up to the standard, or the oliguria time was $<6 \mathrm{~h}$ ), but they have serious electrolyte, acid-base balance disorder and excessive water load, so early administration of CRRT are given for them. The effect of this early administration of CRRT in ECMO patients has not been studied. We here conducted a retrospective analysis of ECMO patients treated by the ECMO team in our hospital, and we preliminarily evaluated the significance of CRRT in the treatment of ECMO patients.

\section{Methods}

\section{General information}

Thirty-two patients who underwent ECMO + CRRT treatment in our hospital from January 2007 to December 2017 were analyzed. There were 22 cases who were male and 10 cases who were female ranging in age from 16 to 69 years. According to the situation at the time of discharge, the patients were divided into the survival group (13 cases) and the death group (19 cases) (Table 1).

\section{Inclusion and exclusion criteria}

\section{Inclusion criteria}

Patients who received CRRT + ECMO treatment were included. And the indicators for using CRRT were: (I) increase in SCr of 1.5-1.9 times baseline; OR increase in sSCr of $\geq 0.3 \mathrm{mg} / \mathrm{dL}(\geq 26.5 \mu \mathrm{mol} / \mathrm{L})$; OR urine output $<0.5 \mathrm{~mL} / \mathrm{kg} / \mathrm{h}$ for $6-12 \mathrm{~h}$ [KDIGO standard established by AKI in 2012 (4)]. (II) ECMO patients who had significant fluid overload; severe hyperkalemia hypernatremia, or both;
Table 1 Diagnosis of primary diseases in patients with ECMO + CRRT

\begin{tabular}{lc}
\hline Primary disease & $\begin{array}{c}\text { Number of patient cases } \\
(\mathrm{n}=32)\end{array}$ \\
\hline Valve implantation & 8 \\
CABG & 3 \\
Pericarditis & 2 \\
Congenital heart disease & 2 \\
Viral myocarditis/stress heart & 7 \\
disease & \\
AMl & 6 \\
Sepsis & 4 \\
\hline
\end{tabular}

ECMO, extracorporeal membrane oxygenation; CRRT, continuous renal replacement therapy; CABG, coronary artery bypass graft; AMI, acute myocardial infarction.

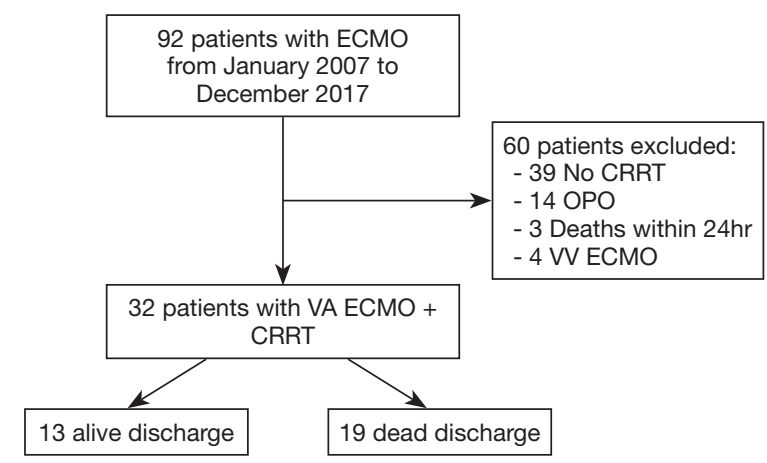

Figure 1 Outcomes of patients with ECMO combined CRRT. ECMO, extracorporeal membrane oxygenation; CRRT, continuous renal replacement therapy; OPO, Organ Procurement Organizations.

other serious electrolyte disturbances; and severe acid-base balance disorders that could not be easily corrected.

\section{Exclusion criteria}

(I) Patients who did not receive CRRT. (II) ECMO patients with organ procurement organization (OPO). (III) Patients who died within $24 \mathrm{~h}$ of CRRT (Figure 1).

\section{CRRT and ECMO connection}

ECMO centrifugal pumps and membrane were from Maquet (Fairfield, NJ, USA). The CRRT was performed using a Prismaflex machine (Gambro, Lund, Sweden). 


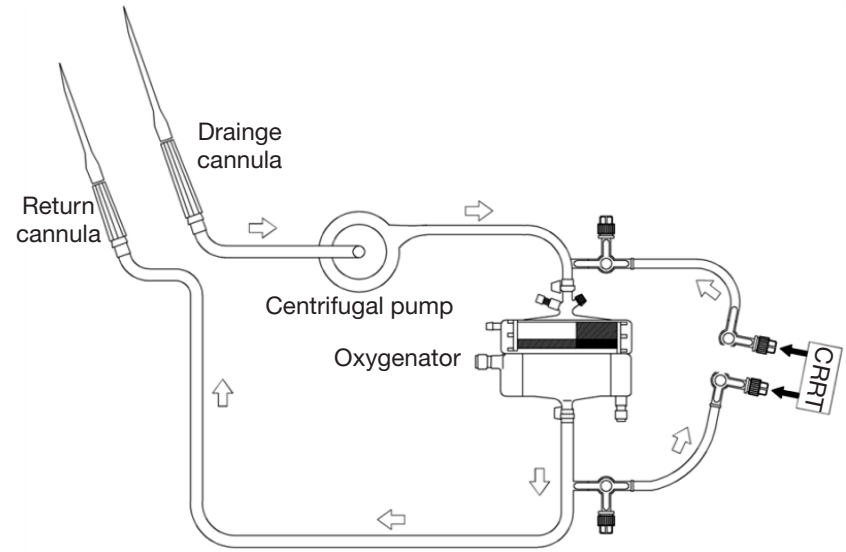

Figure 2 Diagram of ECMO connecting CRRT. ECMO, extracorporeal membrane oxygenation; CRRT, continuous renal replacement therapy.

The connection between CRRT and ECMO is shown in Figure 2 (5). CRRT usually involves CVVH mode, while some patients were given CVVDH mode. The replacement solution was prepared using Port formula. The pre-dilution method maintained the blood flow rate at $150-250 \mathrm{~mL} / \mathrm{h}$ according to the patient's circulation, and the replacement rate was generally $1.5-2.5 \mathrm{~L} / \mathrm{h}$. Body fluid ultrafiltration rate can be adjusted according to the patient's hemodynamics.

\section{Observation parameters}

Statistical analysis was performed on the data of all patients during hospitalization. The gender, age, BMI, ECMO duration, CRRT duration, mechanical ventilation (MV) duration, ICU and hospital length of stay were observed. The fluid balance at ECMO days 1, 3 and 7, clinical biochemical indexes [creatinine, $\mathrm{pH}$, sequential organ failure assessment (SOFA) scores, white blood cell (WBC), bilirubin, and lactate] at CRRT initiation and ECMO weaning in both survival and death groups were also determined.

\section{Statistical analysis}

SPSS 22.0 statistical software was used for statistical analysis. Data were expressed as the frequency and percentage for categorical variables and the mean \pm standard deviation (SD) for continuous variables. The values are presented as the mean $\pm \mathrm{SD}$ and the median and inter quartile range (IQR). All demographic data, clinical data and laboratory findings (non-normally distributed variables) were compared between the two groups (survivors and non-survivors) using the Wilcoxon signed rank test and Mann-Whitney $U$ test for other variables. To compare the proportions of patients, $\chi^{2}$ test or Fisher exact test was performed. Multivariate logistic regression analysis was performed to analyze multiple factors. All statistical tests were two-sided and significance was defined as $\mathrm{P}<0.05$.

\section{Results}

\section{Comparison of clinical data between survival and death groups}

CRRT duration, ECMO to CRRT interval, MV duration, ICU and hospital length were statistically significant between the two groups (all $\mathrm{P}<0.05)$. There was not statistically significant in other factors (all $\mathrm{P}>0.05$ ) between the two groups (Table 2).

\section{Comparison of clinical biochemical indexes affecting patients' survival}

The fluid balance at ECMO day 3, SOFA score and lactate at CRRT initiation, SOFA score at ECMO weaning were statistically significant between the survival and death groups (all $\mathrm{P}<0.05$ ). The fluid balance at ECMO days 1 and 7 , the clinical biochemical indexes (serum creatinine, $\mathrm{pH}, \mathrm{WBC}, \mathrm{Hb}$, bilirubin) at CRRT initiation and ECMO weaning were not statistically significant between the two groups (all $\mathrm{P}>0.05$ ) (Table 3).

\section{Multivariate logistic regression analysis}

We performed a multivariable logistic regression analysis of risk to the prognosis of patients with variables including lactate/SOFA score at CRRT initiation and fluid balance at ECMO day 3. The lactate at CRRT initiation (OR 2.115, 95\% CI: 1.102-4.058, $\mathrm{P}=0.024)$ and fluid balance at ECMO day 3 (OR 5.268, 95\% CI: 1.381-20.088, $\mathrm{P}=0.015$ ) were the independent risk factors to the prognosis of patients.

\section{Discussion}

Patients with severe cardiogenic shock often experience systemic inflammatory reactions and persistent vasospasm and coagulopathy due to low cardiac output or hypoxemia lasting hours or even days prior to ECMO treatment. 
Table 2 Comparison of clinical data between survival and death groups $(\bar{x} \pm \mathrm{s})$

\begin{tabular}{llll}
\hline Factors & Survival group $(\mathrm{n}=13)$ & Death group $(\mathrm{n}=19)$ & $\mathrm{P}$ value \\
\hline Number of male (male, \%) & $8(61.54)$ & $14(73.68)$ & 0.483 \\
Age & $48.00 \pm 14.11$ & $54.84 \pm 6.46$ & 0.073 \\
BMI & $21.34 \pm 1.21$ & $22.16 \pm 1.97$ & 0.189 \\
ECMO duration (h) & $145.23 \pm 20.33$ & $171.74 \pm 45.17$ & 0.057 \\
CRRT duration (h) & $114.69 \pm 27.03$ & $148.37 \pm 48.96$ & 0.032 \\
ECMO to CRRT interval (h) & $5.38 \pm 4.31$ & $10.37 \pm 8.39$ & 0.036 \\
Mechanical ventilation duration (days) & $8.62 \pm 1.76$ & $11.05 \pm 2.80$ & 0.009 \\
ICU length of stay (days) & $10.54 \pm 1.61$ & $15.84 \pm 2.93$ & 0.000 \\
Hospital length of stay (days) & $22.77 \pm 3.54$ & $16.74 \pm 3.38$ & 0.000 \\
\hline
\end{tabular}

Values are presented as median (range) or mean \pm standard deviation (SD). BMI, body mass index.

Table 3 Comparison of clinical biochemical indexes affecting patients' survival

\begin{tabular}{|c|c|c|c|}
\hline Fluid balance and biochemical indexes & Survival group $(n=13)$ & Death group $(n=19)$ & $P$ value \\
\hline Fluid balance at ECMO day 1 & $1,307(1,077-1,600)$ & $1,296(990-1,568)$ & 0.878 \\
\hline Fluid balance at ECMO day 3 & $210(-125$ to 625$)$ & $1,090(750-1,590)$ & 0.000 \\
\hline Fluid balance at ECMO day 7 & $342(360-1,175)$ & $193(-100$ to 400$)$ & 0.773 \\
\hline Serum creatinine at CRRT initiation & $137.31 \pm 51.75$ & $169.89 \pm 50.23$ & 0.085 \\
\hline SOFA score at CRRT initiation & $12.31 \pm 1.93$ & $15.31 \pm 2.36$ & 0.001 \\
\hline WBC at CRRT initiation $\left(\times 10^{9} / \mathrm{L}\right)$ & $12.32 \pm 2.05$ & $12.49 \pm 2.20$ & 0.823 \\
\hline $\mathrm{Hb}$ at $\mathrm{CRRT}$ initiation $(\mathrm{g} / \mathrm{L})$ & $102.23 \pm 11.63$ & $97.00 \pm 8.78$ & 0.157 \\
\hline Bilirubin at CRRT initiation (mmol/L) & $52.77 \pm 21.43$ & $56.42 \pm 30.79$ & 0.714 \\
\hline SOFA score at ECMO weaning & $8.62 \pm 0.77$ & $18.68 \pm 1.38$ & 0.000 \\
\hline WBC at ECMO weaning $\left(\times 10^{9} / \mathrm{L}\right)$ & $11.89 \pm 2.67$ & $12.99 \pm 2.24$ & 0.213 \\
\hline $\mathrm{Hb}$ at ECMO weaning $(\mathrm{g} / \mathrm{L})$ & $103.23 \pm 9.67$ & $99.37 \pm 8.66$ & 0.247 \\
\hline Bilirubin at ECMO weaning $(\mathrm{mmol} / \mathrm{L})$ & $48.77 \pm 23.54$ & $67.21 \pm 41.51$ & 0.125 \\
\hline Lactate at ECMO weaning (mmol/L) & $1.62 \pm 0.54$ & $2.95 \pm 1.31$ & 0.002 \\
\hline
\end{tabular}

Values are presented as median (range) or mean \pm standard deviation (SD). CRRT, continuous renal replacement therapy; SOFA, sequential organ failure assessment; WBC, white blood cell; $\mathrm{Hb}$, hemoglobin.

Both of these factors may lead to multiple organ failure (MOF) (6). Studies have shown that among patients receiving ECMO, more than $30 \%$ have AKI (7), and the prognosis is worse than in those without renal failure.
For this reason, clinical cases of ECMO often require combined treatment with CRRT. Schmidt et al. recently found that $60 \%$ of the 172 adult patients who underwent ECMO were on CRRT, and ECMO combined with CRRT 
Table 4 Multivariate logistic regression analysis of factors affecting patients' survival rate

\begin{tabular}{lll}
\hline Variable & OR $(95 \% \mathrm{Cl})$ & P value \\
\hline Fluid balance at ECMO day 3 & $5.268(1.381-20.088)$ & 0.015 \\
Lactate at CRRT initiation & $2.115(1.102-4.058)$ & 0.024 \\
Constant & 0.001 & 0.014 \\
\hline
\end{tabular}

ECMO, extracorporeal membrane oxygenation; CRRT, continuous renal replacement therapy.

was an independent predictor of 90-day mortality (8). Three studies (9-11) found that hospital mortality was significantly higher in ECMO + CRRT patients than in ECMO alone (OR 5.89, 95\% CI: 4.38-7.92, $\mathrm{P}<0.00001$ ). Even after matching the patient's age, weight, diagnosis, and ECMO patterns, the OR was 6.82 (95\% CI: 4.97-9.36, $\mathrm{P}<0.00001)(12)$. However, there are few studies on the specific factors that affect the mortality of these patients.

Fluid overload is associated with mortality, oxygenation, MV and duration of ICU in critically ill patients. Patients with ECMO are particularly prone to fluid overload, and fluid balance management is often a problem that needs to be addressed (13). In critically ill patients with ECMO, fluid overload leads to worse prognosis (14). Our data also demonstrate that fluid overload at ECMO days 3 and 7 were both higher in non-survivors (Table 3). Radiolabeled isotopes show that the fluid overload is mainly diffusely distributed throughout the total body water and extracellular fluids space during ECMO (15). Continuous accumulation of fluid overload that cannot be cleared increases mortality rate (16) and prolongs ECMO duration (12). These findings are consistent with those of our study (Tables 2 and 3). Fluid overload may reflect the severity of illness. Correction of fluid overload can improve lung function and reduce ECMO weaning time (17). In particular, the incidence of fluid overload in neonatal and child ECMO is higher (18). Gbadegesin et al. reported that the fluid balance in patients with ECMO survivors was lower than that in the death group (19). Symons et al. found that the use of CRRT in ECMO can allow more precise fluid management, which may help reduce the ECMO duration (20). The International ELSO Guidelines recommend 'recovering the extracellular fluid volume to normal (dry weight) and maintaining it' (21). These studies indicate that early CRRT prevention of fluid overload may reduce mortality and improve patient outcome. After adjusting for other significant confounding variables (fluid balance at ECMO day 1, SOFA score at CRRT initiation, lactate at CRRT initiation, SOFA score at ECMO weaning, lactate at ECMO weaning) using logistic regression analysis, an increase in fluid overload on day 3 was found to be an independent predictor of discharge death (Table 4). The increase in fluid volume in the death group at ECMO day 3 may suggest an irreversible decrease in cardiac function or the progressive failure of microcirculation. We have calculated the ECMO to CRRT interval time, and found it to be significantly shorter in the survival group (Table 2). These results suggested that we should use CRRT early, reverse fluid overload, and improve cardiac function.

This study further found that lactate at CRRT initiation in the survival group was significantly lower than that in the death group. Peigh et al. found in a retrospective analysis of 73 patients with VA-ECMO that lactate to be an independent risk factor for the prognosis of patients with ECMO (22). Cheng et al. (23) found that in 145 ECMO patients with or without sepsis, lactate in patients who died at hospital was significantly higher than that of 71 survivors. In 94 patients with pure cardiac VA ECMO, lactate in 1 -year survivors was significantly lower than in those who died (8.6 vs. $2.4 \mathrm{mEq} / \mathrm{L}, \mathrm{P} \leq 0.034)$ (24). This study also found that lactate at CRRT initiation to be an independent risk factor that affected the patient's survival rate. This suggests that microcirculatory perfusion has an impact on mortality. Fluid overload can lead directly to cellular edema and microcirculatory disturbances, which also suggests that early use of CRRT may benefit patients.

While the present study has supplied useful information about ECMO-treated patients, it has several limitations that must be acknowledged. We have included all patients who meet the inclusion and exclusion criteria during the study period for analysis. However, the small patient population and the retrospective nature of the study do not allow us to draw any conclusion about the effectiveness of combined ECMO-CRRT treatment. According to the clinical control test (the test level is 0.05 ), the accuracy of this study was $80 \%$. Larger series from multi-center RCT experiments are needed to confirm the effectiveness of the study and to provide a reliable theoretical basis for followup clinical work.

In summary, our results suggest that lactate at CRRT initiation and fluid balance at ECMO day 3 are the independent risk factors of prognosis for patients on ECMO. The main indicator of evaluation was fluid balance at ECMO day 3, which were 210 and $1,090 \mathrm{~mL}$ in the death and survival groups. If CRRT device is introduced as soon 
as possible into the ECMO circuit, it may be possible to promote early fluid balance and improve patient survival.

\section{Acknowledgements}

Funding: This study was supported by the Hospital Foundation (SWH2016ZDCX2007 and SWH2016JSTSYB-51).

\section{Footnote}

Conflicts of Interest: The authors have no conflicts of interest to declare.

Ethical Statement: The study was approved by the Ethical Committee of Southwest Hospital (the number/ID of the approval is KY 201847).

\section{References}

1. Kielstein JT, Heiden AM, Beutel G, et al. Renal function and survival in 200 patients undergoing ECMO therapy. Nephrol Dial Transplant 2013;28:86-90.

2. Wu MY, Lin PJ, Tsai FC, et al. Impact of preexisting organ dysfunction on extracoporeal life support for non -postcardiotomy cardiopulmonary failure. Resuscitation 2008;79:54-60.

3. Lan C, Tsai PR, Chen YS, et al. Prognostic factors for adult patients receiving extracorporeal membrane oxygenation as mechanical circulatory support a 14-year experience at a medical center. Artif Organs 2010;34:E59-64.

4. Khwaja A. KDIGO clinical practice guidelines for acute kidney injury. Nephron Clin Pract. 2012;120:c179-84.

5. Seczyńska B, Królikowski W, Nowak I, et al. Continuous renal replacement therapy during extracorporeal membrane oxygenation in patients treated in medical intensive care unit: technical considerations. Ther Apher Dial 2014;18:523-34.

6. Hei F, Lou S, Li J, et al. Five-year results of 121 consecutive patients treated with extracorporeal membrane oxygenation at Fu Wai Hospital. Artif Organs 2011;35:572-8.

7. Aubron C, Cheng AC, Pilcher D, et al. Factors associated with outcomes of patients on extracorporeal membrane oxygenation support: a 5 -year cohort study. Crit Care 2013;17:R73.

8. Schmidt M, Bailey M, Kelly J, et al. Impact of fluid balance on outcome of adult patients treated with extracorporeal membrane oxygenation. Intensive Care
Med 2014;40:1256-66.

9. Paden ML, Warshaw BL, Heard ML, et al. Recovery of renal function and survival after continuous renal replacement therapy during extracorporeal membrane oxygenation. Pediatr Crit Care Med 2011;12:153-8.

10. Ricci Z, Morelli S, Favia I, et al. Neutrophil gelatinaseassociated lipocalin levels during extracorporeal membrane oxygenation in critically ill children with congenital heart disease: preliminary experience. Pediatr Crit Care Med 2012;13:e51-4.

11. Wolf MJ, Chanani NK, Heard ML, et al. Early renal replacement therapy during pediatric cardiac extracorporeal support increases mortality. Ann Thorac Surg 2013;96:917-22.

12. Blijdorp K, Cransberg K, Wildschut ED, et al. Haemofiltration in newborns treated with extracorporeal membrane oxygenation: a case-comparison study. Crit Care 2009;13:R48.

13. Selewski DT, Cornell TT, Blatt NB, et al. Fluid overload and fluid removal in pediatric patients on extracorporeal membrane oxygenation requiring continuous renal replacement therapy. Crit Care Med 2012;40:2694-9.

14. Fleming GM, Askenazi DJ, Bridges BC. A multicenter international survey of renal supportive therapy during ECMO: The Kidney Intervention During Extracorporeal Membrane Oxygenation (KIDMO) group. ASAIO J 2012;58:407-14.

15. Anderson HL, Coran AG, Drongowski RA, et al. Extracellular fluid and total body water changes in neonates undergoing extracorporeal membrane oxygenation. J Pediatr Surg 1992;27:1003-7.

16. Selewski DT, Cornell TT, Lombel RM, et al. Weightbased determination of fluid overload status and mortality in pediatric intensive care unit patients requiring continuous renal replacement therapy. Intensive Care Med 2011;37:1166-73.

17. Askenazi DJ, Selewski DT, Paden ML, et al. Renal replacement therapy in critically ill patients receiving extracorporeal membrane oxygenation. Clin J Am Soc Nephrol 2012;7:1328-36.

18. Hoover NG, Heard M, Reid C, et al. Enhanced fluid management with continuous venovenous hemofiltration in pediatric respiratory failure patients receiving extracorporeal membrane oxygenation support. Intensive Care Med 2008;34:2241-7.

19. Gbadegesin R, Zhao S, Charpie J, et al. Significance of hemolysis on extracorporeal life support after cardiac surgery in children. Pediatr Nephrol 2009;24:589-95. 
20. Symons JM, McMahon MW, Karamlou T, et al. Continuous renal replacement therapy with an automated monitor is superior to a free-flow system during extracorporeal life support. Pediatr Crit Care Med 2013;14:e404-8.

21. Extracorporeal Life Support Organization. Guidelines. Available online: https://www.elso.org/Resources/ Guidelines.aspx. Accessed March 8, 2012.

22. Peigh G, Cavarocchi N, Keith SW, et al. Simple new risk score model for adult cardiac extracorporeal membrane

Cite this article as: $\mathrm{He} \mathrm{P}$, Zhang $\mathrm{S}, \mathrm{Hu} \mathrm{B}, \mathrm{Wu}$ W. Retrospective study on the effects of the prognosis of patients treated with extracorporeal membrane oxygenation combined with continuous renal replacement therapy. Ann Transl Med 2018;6(23):455. doi: 10.21037/atm.2018.11.12 oxygenation: simple cardiac ECMO score. J Surg Res 2015;198:273-9.

23. Cheng A, Sun HY, Lee CW, et al. Survival of septic adults compared with nonseptic adults receiving extracorporeal membrane oxygenation for cardiopulmonary failure: A propensity-matched analysis. J Crit Care 2013;28:532.e1-10.

24. Byku M, Piotrowski R, Itoh A, et al. Pre-Extra Corporeal Membrane Oxygenation (ECMO) MELD Score May Predict Meaningful Survival. J Heart Lung Transpl 2016;35:S380-1. 